\title{
Research on Traffic Planning Modes Based on Transport System Characteristics of Linear City
}

\author{
Lingyan Cheng ${ }^{a}$, Shiwei $\mathrm{He}^{\mathrm{b}}$ \\ School of Traffic and Transportation, Beijing Jiaotong University, Beijing 100044, China \\ azjlycheng@163.com, bshiweihe@bjtu.edu.cn
}

\begin{abstract}
Faced with the growing problem of urban congestion, the priority to develop public transport has been thought to ease congestion effect [1]. For linear cities, there is some unique characteristics. Wide transverse and narrow longitude lead to the difference from other cities of single-center circle style in the same scale. Urban form has an important impact on urban land structure, urban transport characteristics, and urban road network structure and so on. These factors are important factors in the city's public transportation network planning, and have a decisive impact on the layout of urban public transportation network. After studying the characteristics of urban transport, this paper discusses the transportation system planning based on problems and requirements of urban transport system by linear city form. Traffic planning mode can be mainly divided into public transit hub planning and route network layout planning of linear city.
\end{abstract}

Keywords: linear city; traffic planning; transport characteristics; planning modes.

\section{Traffic characteristics of linear city}

\subsection{Basic characteristics of urban transport}

Road network of linear city is formed by radial or chained Trunk road. Road network system generally includes through traffic, arterial road across group and inner group road network system. The basic function of the through road system is to divert through traffic quickly, separate through traffic and urban traffic and improve the outside traffic gateway. Inner group road network system assumes the life and service traffic in group [2]. Combined linear city are generally advocating transit-oriented urban development patterns with group nodes. This is due to the limit of lateral width and valuable space resources in longitudinal section, which is also an important factor to select public transport mode for linear cities.

Larger linear cities always set up BRT (or light rail) to strengthen the links between various decentralized urban areas [3]. The medium-sized cities will develop bus system as the main form. In addition, it also set expressway in the city arterial road, section lines in the group to form centralized bus network.

\subsection{Characteristics of road network}

Formation of linear cities is always due to geographical factors. Most of the road network structure looks like herringbone between major groups. Different structures are adopted by road network within group depending on the group function. In general, road network of linear city is consisted of one or several major trunk road or freeway as traffic axis. It connects large and medium-sized groups and internal road network like a spine. Specifically, the characteristics can be summed up as follows:

(1)Blurry location of road network system function

Urban road network system consists of through roads, arterial road and others. Through roads are expressway of communication of cities [4]. It is also committed to the separation of through traffic and unban traffic and the improvement of urban external traffic interface [5]. Arterial road assumes the life and service traffic in cities' groups. Simultaneously, high land use density of linear city results in the blurry boundary of through roads and road network within groups.

(2)Road network of linear city is supported by several major trunk roads in main development axis direction, which connects each groups as linear structure.

From the overall macro perspective, the skeleton of road network of linear city is consist of major trunk roads in main development axis direction, which links the groups across the two ends of line. 
Groups and the main trunk road look like bead. Generally, main trunk roads are parallel to each other along the river channel and other natural restrictions.

(3)There are differences between road network structures within groups. Internal road network of each group has its own characteristics and flow into main roads. Road network within groups has the advantages of high density and accessibility.

(4)Road network density between groups is small and compactness of city is low

Due to the low compactness of linear city, linear cities are longer than cities of the same size. In the meanwhile, longer distance between city groups and worse city development between groups are also big problems. Road network is mainly consist of main trunk roads and a few feeder. There is only a little traffic interface between inside and outside groups. Therefore, road network structural imbalances between groups and within groups. And integration of road network is in a low degree.

\subsection{Resident trip characteristics of linear city}

Resident Trip Characteristics of linear city has the particularity of restrictions on its urban form, which determines the significant differences of trip characteristics to others with urban forms. By comparing the most two common city forms, single-center circle style and linear city, this paper analyzed resident trip characteristics of linear city.

Resident trip frequency

Longer trip distance and time of resident trip caused by significant long axis of linear city also result in the fewer number of trips. Resident per capita trip times of linear cities is universally less than cities of single-center circle style in the same scale [6].

Resident trip mode

Different modes of transport, especially their reasonable travel distance, are decided by the dynamic economic characteristics of transport mode. Resident average travel distance increases due to the long transverse traffic axis of linear city, which also lead to the development of motorization. Walking or cycling will be converted to motorcycles, public transport and private vehicles. However, it will increase the contribution rate of walking because of the shorter longitudinal distance traffic of linear cities. Rate of public transport and walking of linear cities is obviously higher than cities of single-center circle style in the same scale.

Resident trip distance

Both ends of the group of communication is more difficult because linear cities develop along the transverse traffic axis. Although longitudinal transportation distance of linear cities is short, resident per capita trip distance is longer than cities of single-center circle style about $15 \%-30 \%$. And it will increase with the increase of city size.

\section{Requirements of urban transport system by linear city form}

Modern cities need to establish an integrated transport system network mode, including road network mode, light rail development mode and bus network structure mode, to meet the needs of urban transport and urban space layout requirements. Due to limits of the transverse width of linear city, consequently, its transverse traffic resource is relatively limited. The Government should take full advantage of the guidance function of traffic to the form of urban land use layout. For new urban area development plan, we should choose a reasonable mode of transport , in order to guide the gradual implementation of detailed traffic system spatial layout plan, and planning control of transportation facilities land during construction.

\section{Traffic problems of linear city}

Linear city has general problems of urban roads, such as low road network density, blended function of urban roads, inadequate branch road, imbalance of the structural proportion of trunk and branches and so on. These also result in the aggregation of long-distance through traffic, block traffic, motor vehicle and non-motor vehicles on the city trunk roads. Fast and slow traffic is so mixed to cause the low traffic efficiency. Urban road network of low density also makes the city bus line is too 
concentrated. Meanwhile, it may bring small rate of bus service coverage and poor service levels. Thereby it stimulates the growth of private motor vehicles. The determining of urban roads function is beneficial to the performance of urban road system and better life space. As the city main road, longitudinal roads of linear city support the inner and through traffic. However, intersections will be formed with transverse roads, inevitably. If grade separations are laid too much, the cost will be too high. On the contrary, if there is only grade crossing, it's harmful to gather and evacuate in intersections. Many traffic problem has become a constraint to further development of city and one of the problems to be solved, especially in big cities and megacities.

\section{Urban transport planning mode}

Since linear cities' unique spatial form and traffic characteristics, its dependence on public transportation should support and guide the TOD mode [7], promote the conversion to public transport-dependent in city development. The proposed public transport planning mode of linear city is mainly divided into public transit hub planning and route network layout planning.

\section{1 public transit hub planning of linear city}

From the perspective of public transport planning [8], public transit hub is a place for transformation of traffic function, is the interchange point of passenger flow of different modes and different directions. Therefore, as the critical infrastructure in the public transport system, transfer hub will be set to be diversion between land use and traffic demand.

\section{2 route network layout planning of linear city}

Combined with the general urban public transportation network layout method and current bus situation, city bus lines planning can be summarized as two modes [9]. The first mode still adopt the line network layout scheme mainly focused on radial center depending on the volume, flow and trip habits of bus passengers. In addition, mode one only adjust, enrich and improve on the basis of the existing public transportation network, but no more clear distinction on the difference of service area and service object. The other mode uses the route network layout of radiation plus ring. Because the structural characteristics of linear city is longitudinal bus lines of long-distance. Furthermore, since the development of the area, bus lines will be stretched. Through the construction of public transit transfer hub facilities, external radiation line can be blocked at the edge of the city. At the same time, it is convenient for residents to take bus into city center through transfers. This would solve the current problem of unclear function of urban lines in and around city center, low repetition rate and efficiency.

\section{References}

[1]. Zhibin Zhang, Meijuan Jin. City Planning and City Space Structure Modelling--A Case Study of Shenzhen City [J]. JOURNAL OF NORTHWEST NORMAL UNIVERSITY (SOCIAL SCIENCES), 2003, 06:85-88.

[2]. Yichun Wang, Shusheng Wang. Traffic feature analysis and ecological transport planning practice of linear city_ a case study of Aletai center[A]. Urban Planning Society of China. City Times, Collaborative Planning__2013 China Urban Planning Symposium(01- Urban Roads and Transport Planning)[C]. Urban Planning Society of China:, 2013: 9.

[3]. Yifang Luo. Research on the urban design of North River area based on Xining city's overall development strategy [D]. Xi'an University of Architecture and Technology, 2012.

[4]. Ruiwei Chen, Qiyuan Peng. Enlightenments from the Parallel Local/Express Lines of New York Subway for Urban Rail Network of the Ribbon-form Cities [J].Journal of Transportation Engineering and Information, 2013, 02:70-76.

[5]. Yixin Zhao, Qing Ma, Jingyan Fu, et al. Deconstruction of Qingdao Urban Space from the Respective of Transportation [J]. City Planning Review, 2014, 02:37-41.

[6]. Jianguo Xin. Research on the Urban Design of North Area Based on Xining city's Overall Development Strategy. [D] Xi'an University of Architecture and Technology, 2009. 
[7]. Qiong Wang. Study on the Line Network Planning Methods of Bus Rapid Transit of Linear City Based on Transit-Oriented-Devolopment[D]. Lanzhou Jiaotong University, 2013.

[8]. Dezhao Hou, Miaoxi Zhao, Kefei Yan. A Study on the Application of the Pole-corridor Mode in Belt City Transportation Planning__ A Case Study of Dandong Economic Zone [J]. PLANNERS, 2007, 09:89-93.

[9]. Kai Wang. Research on the Public Transit Network Planning of Small and Medium Linear Cities [D]. Chang'an University, 2012. 